\section{Tapering without relapse in rheumatoid arthritis patients with high TNF blocker concentrations: data from STRASS study}

We read with interest the paper by l'Ami $e t$ al ${ }^{1}$ reporting the safety of a single step-down strategy without flare-up of disease in rheumatoid arthritis (RA) patients treated with adalimumab associated with high trough concentrations. At the time of personalised medicine, prediction of the absence of relapse during tapering strategy is a huge challenge to improve this approach. Furthermore, EULAR recommendations proposed in RA patients in remission without glucocorticoids to first step down the bDMARDs. ${ }^{2}$ So, we investigated the interest of TNF blocker blood concentration assessment in order to predict the absence of relapse during tapering in the STRASS study. ${ }^{3}$ The STRASS study demonstrated the feasibility of step-down therapeutic strategy compared with maintenance strategy in RA patients in clinical remission treated with adalimumab or etanercept. In contrast to l'Ami study, which performed a single tapering, successive tapering step every 3 months in RA patient still in remission was performed. Among the 137 patients included in STRASS study, 132 serum samples were collected solely at baseline without other blood collections and assessed by ELISA with Lisa Tracker (adalimumab or etanercept kit by Theradiag, Marne-La-Vallee, France). We defined high level of TNF blocker, by concentration higher or equal to upper detection limits in serums $(8 \mu \mathrm{g} / \mathrm{mL}$ for adalimumab and $5 \mu \mathrm{g} / \mathrm{mL}$ for etanercept). For adalimumab, this definition was similar to the definition of high trough concentrations defined by l'Ami.

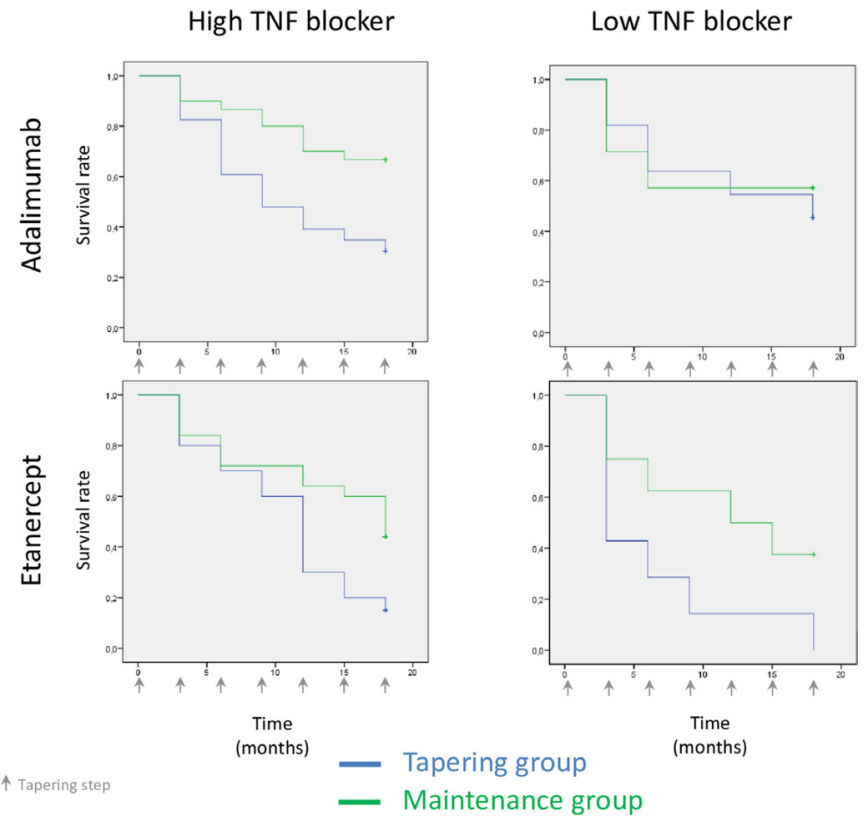

Figure 1 Survival without relapse according to TNF blockers trough concentration. Survival without relapse was the same between tapering or maintenance group in case of high TNF blocker trough concentration at baseline during the first 3 months and almost the same over 9 months for etanercept. However, the survival rate in the tapering group strongly dropped after 6 months for adalimumab. This was in accordance to I'Ami study since adalimumab trough concentration decreased under the high concentration threefold at 12 and 24 weeks after only one tapering.

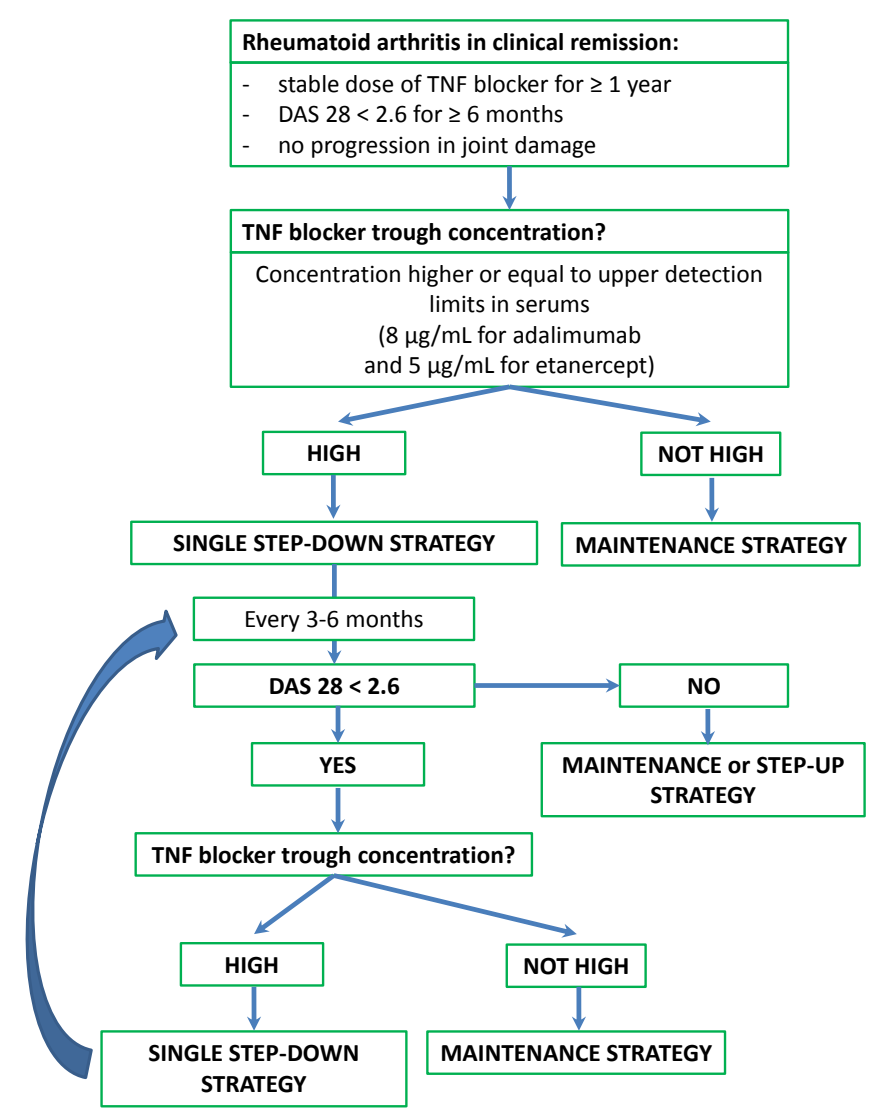

Figure 2 Proposed algorithm based on therapeutic drug monitoring to improve tapering strategy.

Overall, in STRASS study, no clear effect was observed between high blood levels of TNF blockers at baseline and persistence of remission over 24 months. However, when focusing at 6 months (that means two first steps-down in the spacing arm), the proportion of patients without relapse was higher in case of high TNF blockers concentration at baseline $\left(\chi^{2}=6.22 ; p=0.01\right.$; figure 1$)$. In the l'Ami study, adalimumab trough concentration decreased under the high concentration level at 12 and 24 weeks after only one tapering. This could explain the increased rate of relapse after the third tapering in STRASS (figure 1). Furthermore, no data with etanercept on tapering are available to date. Difference pattern of flares between RA patients treated by adalimumab or etanercept could be due to the absence of cut-off previously reported for etanercept.

Our data suggest to perform a drug monitoring before each tapering, in order to avoid the situation with low TNF blockers blood trough concentration leading to clinical relapse. Furthermore, to reduce the high TNF blockers trough concentration could be also benefit for the RA patients in remission since high TNF blockers trough concentration was reported to be associated with a strong risk of infection. ${ }^{4}$

The clinical utility of TNF blockers monitoring and determination of specific cut-offs in predicting clinical remission had already been explored especially in inflammatory bowel diseases. ${ }^{5}$ Here, we claim the monitoring of trough concentrations in order to improve successful tapering strategy (figure 2).

In conclusion, we confirmed that tapering is feasible without an increased rate of relapse in RA patients with 
clinical remission and high TNF blocker blood concentration. Furthermore, since the initial concentration of STRASS study will be predictive of RA relapse in case of TNF blocker injection spacing, we propose to assess trough TNF blocker concentration before each tapering step in order to maintain remission and avoid a relapse in RA patients with clinical remission. Finally, we proposed an algorithm to manage step-down strategy (figure 2), which should be confirmed in a prospective study.

\section{Hubert Marotte $\odot{ }^{1,2}$ Mélanie Rinaudo-Gaujous, ${ }^{3}$ Cécile Petiet, ${ }^{1}$} Bruno Fautrel, ${ }^{4,5}$ Stéphane Paul ${ }^{6}{ }^{6}$

${ }^{1}$ Rhumatologie, CHU Saint-Etienne, Saint-Etienne, France

${ }^{2}$ SAINBIOSE, INSERM U1059, University of Lyon, Saint-Etienne, France

${ }^{3} \mathrm{CHU}$ Saint-Etienne, Laboratory of Immunology and Immunomonitoring, SaintEtienne, France

${ }^{4}$ Rheumatology, Assistance Publique-Hopitaux de Paris, Paris, France

${ }^{5}$ GRC08_-IPLESP, UPMC Faculte de Medecine, Paris, France

${ }^{6}$ Laboratory of Immunology and Immunomonitoring, Universite Jean Monnet SaintEtienne Faculte de Medecine Jacques Lisfranc, Saint Priest en Jarez, France

Correspondence to Professor Hubert Marotte, Rhumatologie, CHU Saint-Etienne, Saint-Etienne 42270, France; hubert.marotte@chu-st-etienne.fr

Contributors HM, MR-G, BF and SP designed the study. MR-G, CP and SP performed the experiments. HM, MR-G, CP, BF and SP analysed the data. $\mathrm{HM}, \mathrm{CP}, \mathrm{BF}$ and SP wrote the draft of the manuscript, which was revised by all the authors. The final version was approved by all the authors.

Funding This work was funded by the AbbVie.

Patient consent for publication Obtained.

Provenance and peer review Not commissioned; internally peer reviewed.

(c) Author(s) (or their employer(s)) 2020. No commercial re-use. See rights and permissions. Published by BMJ.

\section{Check for updates}

To cite Marotte H, Rinaudo-Gaujous M, Petiet C, et al. Ann Rheum Dis 2020;79:e81.

Received 13 April 2019

Accepted 17 April 2019

Published Online First 2 May 2019

\section{SLinked}

- http://dx.doi.org/10.1136/annrheumdis-2019-215609

Ann Rheum Dis 2020;79:e81. doi:10.1136/annrheumdis-2019-215546

\section{ORCID iDs}

Hubert Marotte http://orcid.org/0000-0003-1177-9497

Stéphane Paul http://orcid.org/0000-0002-8830-4273

\section{REFERENCES}

1 I'Ami MJ, Krieckaert CL, Nurmohamed MT, et al. Successful reduction of overexposure in patients with rheumatoid arthritis with high serum adalimumab concentrations: an open-label, non-inferiority, randomised clinical trial. Ann Rheum Dis 2018;77:484-7.

2 Smolen JS, Landewé R, Bijlsma J, et al. EULAR recommendations for the management of rheumatoid arthritis with synthetic and biological disease-modifying antirheumatic drugs: 2016 update. Ann Rheum Dis 2017;76:960-77.

3 Fautrel B, Pham T, Alfaiate T, et al. Step-down strategy of spacing TNF-blocker injections for established rheumatoid arthritis in remission: results of the multicentre noninferiority randomised open-label controlled trial (STRASS: spacing of TNF-blocker injections in rheumatoid arthritis study). Ann Rheum Dis 2016;75:59-67.

4 Bejan-Angoulvant T, Ternant D, Daoued F, et al. Brief report: relationship between serum infliximab concentrations and risk of infections in patients treated for spondyloarthritis. Arthritis Rheumatol 2017;69:108-13.

5 Paul S, Del Tedesco E, Marotte H, et al. Therapeutic drug monitoring of infliximab and mucosal healing in inflammatory bowel disease: a prospective study. Inflamm Bowel Dis 2013;19:2568-76 